\title{
COHESIVE ZONE BASED CONFORMAL MESHING AND DAMAGE SIMULATION OF YARN CONTACTS IN WOVEN COMPOSITES
}

\author{
Anqi Li ${ }^{1,2, *}$, Badadjida Wintiba ${ }^{1}$, Joris J. C. Remmers ${ }^{2}$, Marc G. D. Geers ${ }^{2}$, Thierry J. \\ Massart $^{1}$ \\ ${ }^{1}$ Universite libre de Bruxelles (ULB), 1050 Brussels, Belgium \\ ${ }^{2}$ Eindhoven University of Technology (TU/e), 5600 MB, Eindhoven, The Netherlands \\ *anqi.li@ulb.be
}

The mechanical response of woven composites under off-axis tensile loading is closely governed by the damage mechanism in the yarn-yarn/matrix interfaces. For off-axis tensile loading, the yarns will reorient towards the loading direction and lead to a rather ductile behavior after the decohesion of the yarn contact interfaces. Recent works [1] have proposed an automated methodology to generate a RVE geometry based on implicit descriptions based on distance fields, resulting in geometries free of yarn interpenetration by inserting a gap between yarns to enable a conformal discretization [2]. However, this leads to high computational costs due to refined elements in the gap region during the simulations. This contribution extends this methodology to extract the geometry of contacting surfaces from the distance fields which can be subsequently used in a conformal meshing strategy for the RVE with cohesive zone elements (CZE). A mesh comparison between the methodology proposed in [1] and the current methodology is illustrated by a plain weave RVE in Figure 1. The numerical simulation of the plain weave RVE with shear loading in ABAQUS and the load displacement curves are also presented in Figure 1 in which a softening response can be reproduced due to the damage of CZE in interface and plasticity in matrix.
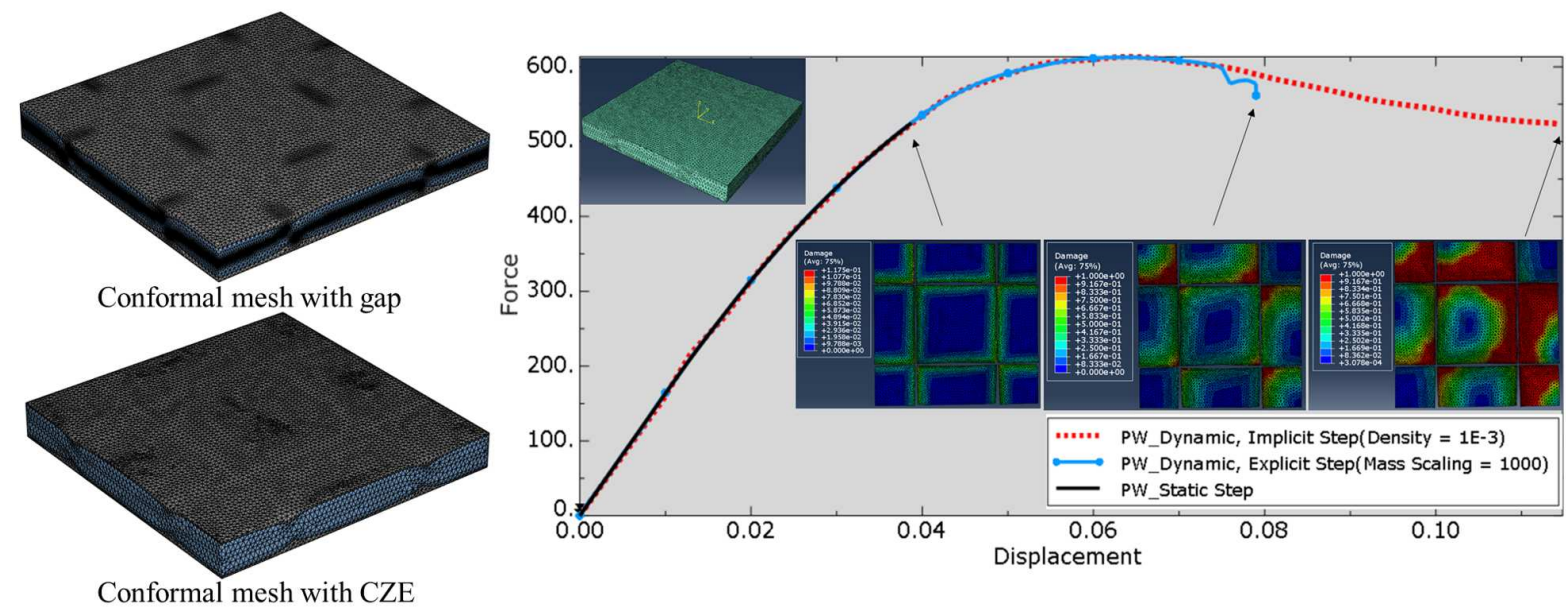

Figure 1: A conformal mesh for a plain weave RVE with gap or with CZE in the interface (Left). Load-displacement curve of the RVE with CZE under shear loading in ABAQUS (Right)

\section{References}

[1] Wintiba B., Sonon B., Ehab Moustafa Kamel K., Massart T. J., An automated procedure for the generation and conformal discretization of 3D woven composites RVEs.Composite structures(2017), 180, 955-971.

[2] Ehab Moustafa Kamel K., Sonon B., Massart T. J., An integrated approach for the conformal discretization of complex inclusion-based microstructures, Computational Mechanics(2019), 64, 1049. 